\title{
Creencias epistemológicas generales, académicas y disciplinares en relación con el contexto*
}

\author{
General, Academic and Domain-Specific Epistemological \\ Beliefs in Connection with Context
}

Recibido: junio 3 de 2009 ～Revisado: septiembre 10 de 2009 | Aceptado: octubre 2 de 2009

\author{
FRANCISCO LEAL-SOTO** \\ Universidad de Tarapacá, Chile
}

Para citar este artículo. Leal-Soto, F. (2010). Creencias epistemológicas generales, académicas y disciplinares en relación con el contexto. Universitas Psychologica, 9 (2), 381-392.

* Esta investigación ha sido realizada con apoyo del Fondo Nacional para el Desarrollo de la Ciencia y la Tecnología del Gobierno de Chile, a través del proyecto FONDECYT 1061282. Reconocimientos: El autor desea agradecer a Jimmy Ávalos, Carlos Espinoza, Marco Iraola, Juan Malebrán, Mónica Miranda y Elda Pérez, por su colaboración en la recolección de los datos..

** Avda. Luis Emilio Recabarren 2477, Iquique, Chile. Correo electrónico : fleal@uta.cl

\begin{abstract}
RESUMEN
Las creencias epistemológicas tendrían un carácter multinivel: general, académico y disciplinar. Sin embargo, el contexto ha sido considerado de manera general y poco precisa. El objetivo de este estudio fue establecer diferencias en la comprensión epistemológica a través de los niveles, considerando el rol del contexto. En un diseño intrasujetos, se evaluó la comprensión epistemológica en cada uno de los tres niveles, en contexto pedagógico y en contexto social-informal, a cuatro grupos de 85 participantes con distinta proximidad al contexto pedagógico. Los resultados confirman diferencias entre creencias en los niveles general y académico y general o disciplinar, pero no entre los niveles académico y disciplinar. La influencia del contexto y la proximidad al mismo fueron confirmadas, pero de manera inesperada. Palabras clave autores

Creencias epistemológicas, generalidad-especificidad, contexto.

Palabras clave descriptores

Creencias epistemológicas, rendimiento académico, pedagogía, investigaciones.
\end{abstract}

\begin{abstract}
A B S T R A C T
Multilayer nature in personal epistemology, with general, academic and domain levels of epistemological beliefs has been proposed. However, context has been considered in general and imprecise way. The aim of this study was to establish differences across epistemological beliefs levels, considering the influence of context. In an intrasubject design, epistemological understanding in each of the three levels was assessed in specialized and non-specialized context, on four groups of participants who have different proximity with specialized context. Results support differences across levels of beliefs, but only among general and academic and domain beliefs, not among academic and domain beliefs. Context nature and proximity with context were also relevant, but in unexpected way.

Key words authors

Epistemological Beliefs, Generality-Specificity, Context.

Key words plus

Epistemological Beliefs, Academic Achievement, Teaching-Research.
\end{abstract}




\section{Introducción}

Epistemología personal es un término que hace referencia a las creencias que las personas sostienen acerca del conocimiento, tanto respecto de su naturaleza como de la adquisición y justificación del mismo (Hofer, 2002). Aunque el concepto de creencias resulta difuso (Pajares, 1992) e incluso podría superponerse con el concepto de conocimiento (Alexander \& Dochy, 1994; Alexander, Murphy, Guan \& Murphy, 1998), hay consenso en que se distinguen de éste por su mayor compromiso afectivo, su falta de apego a la lógica, su resistencia al cambio y su fuerte influencia sobre la conducta (Schommer-Aikins, 2004). Pajares también destaca que las creencias no requieren consenso grupal, constituyéndose en certezas subjetivas, a diferencia del conocimiento, una de cuyas características sería la validación o consenso grupal.

Las creencias epistemológicas han sido motivo de una creciente preocupación en la Psicología Educacional en las últimas décadas, como se aprecia en una rápida mirada a la literatura especializada (p. e., Bendixen \& Rule, 2004; Brownlee \& Berthelsen, 2006; Hofer, 2004a; Hofer \& Pintrich, 1997; Hofer \& Pintrich, 2002; Limón, 2006a; Muis, Bendixen \& Haerle, 2006). La preocupación por la epistemología personal ha surgido con fuerza luego de que los fracasos de los procesos de reforma educativa llevados adelante en la mayor parte de los países pusieran de relieve el papel de las creencias de los profesores tanto para el éxito de los procesos de reforma como de los propios procesos de aprendizaje (Calderhead, 1996). Entender cómo piensan los profesores acerca del conocimiento y el aprendizaje puede contribuir a desarrollar acciones que faciliten su adscripción a los modelos curriculares y metodológicos necesarios para adecuar los procesos educativos a las necesidades formativas que plantea la emergente realidad del mundo globalizado de la era de la información (Delors, 1996).

Aunque diferentes líneas de investigación han conducido a varios modelos teóricos, la mayor parte de ellos comparten las líneas gruesas y contribuyen a generar un marco coherente para la investigación en epistemología personal, pero tam- bién comparten serias debilidades y confusiones (Chandler, Hallett \& Sokol, 2002; Hofer, 2002; Hofer \& Pintrich, 1997; Limón, 2006a; Schraw \& Sinatra, 2004; Pintrich, 2002). Uno de estos tópicos confusos ha sido el de la generalidad o especificidad de las creencias epistemológicas a través de las disciplinas o dominios de conocimiento, que ha sido recientemente debatido en forma intensa y profunda (Buehl \& Alexander, 2006; Hofer, 2006; Limón, 2006a, 2006b; Muis, Bendixen \& Haerle, 2006) y que se relaciona con el tema de su contextualización, asunto mucho más descuidado en la literatura (Hofer, 2006; Muis, 2004).

La generalidad a través de disciplinas ha sido inicialmente reclamada a partir de dos estudios diseñados específicamente para probarla (Schommer \& Walker, 1995). Usando el Cuestionario Epistemológico de Schommer modificado para direccionarlo hacia dos disciplinas -Ciencias Sociales y Matemáticas-, estos investigadores evaluaron repetidamente a dos grupos de sujetos con ambos instrumentos, incluyendo una prueba de comprensión de lectura en uno de ambos dominios. Correlacionaron las creencias entre disciplinas, y compararon el poder predictivo de las creencias en ambos dominios respecto de la comprensión lectora del texto específico de dominio. Los resultados mostraron correlaciones moderadas entre los dominios, y capacidades predictivas similares de las creencias en ambas disciplinas sobre la comprensión lectora, con independencia del dominio del texto. Interpretan estos resultados como un soporte para la hipótesis de que las creencias epistemológicas son generales e independientes de la disciplina.

Buehl, Alexander y Murphy (2002) se propusieron examinar la hipótesis de la especificidad por dominio con mayor precisión que en el estudio de Schommer y Walker (1995), ya que, a pesar del direccionamiento del instrumento utilizado en ese estudio hacia disciplinas específicas, el instrumento fue el mismo que el utilizado para evaluar las creencias generales. Para ello, diseñaron instrumentos específicos para los dominios de Matemáticas e Historia, equivalentes entre sí. Con estos instrumentos, encontraron que los estudiantes sostienen 
creencias diferentes respecto de ambas disciplinas. A partir de este resultado, las autoras descartan la posibilidad de una estructura generalizada a través de los dominios; sin embargo, argumentan que probablemente las creencias epistemológicas sean en algún grado específicas y en algún grado generales, como Sternberg (1989 en Buehl, Alexander $\&$ Murphy, 2002) ha propuesto respecto de la inteligencia. Sostienen que los resultados diferentes obtenidos en diversos estudios se deben, probablemente, a la orientación de los instrumentos utilizados en cada uno de ellos: los realizados con instrumentos orientados generalmente, arrojan datos consistentes con la generalidad de las creencias, mientras que los orientados específicamente, como en el caso de su propio estudio, arrojan datos consistentes con la especificidad de las mismas.

Buehl y Alexander (2001) propusieron que las creencias epistemológicas pueden ser comprendidas y evaluadas en tres niveles anidados: creencias epistemológicas generales, que se evidencian cuando las preguntas son amplias, y muestran una progresión consistente en los individuos; creencias acerca del conocimiento académico o escolar, nivel que postulan como necesario debido a la especificidad del conocimiento vinculado a la escolarización (Alexander \& Murphy, 1998; Buehl \& Alexander, 2006) y creencias específicas de dominio, que surgen cuando las preguntas son más acotadas. En una reciente reformulación del modelo (Buehl \& Alexander, 2006), estas autoras distinguen entre los niveles general y disciplinar de creencias epistemológicas, descartando el nivel académico como un nivel específico distinto del disciplinar, aunque proponen que las creencias a nivel disciplinar se derivarían progresivamente desde las creencias generales, según avanza la escolarización.

Como resultado de su propia revisión de la literatura al respecto, Muis, Bendixen y Haerle (2006) proponen un modelo en el que conservan la estructura de tres niveles, pero también asumen que la importancia relativa de las creencias en estos niveles sería variable, siendo inicialmente más importantes las generales, para ir perdiendo importancia ante las académicas con el progreso de la escolarización, hasta llegar a ser más rele- vantes las disciplinares en niveles avanzados de escolarización.

Junto con avanzar en la comprensión de las creencias epistemológicas, se ha hecho cada vez más necesario entender la relación entre la epistemología personal y el contexto. Schommer-Aikins, por ejemplo, destaca que las creencias epistemológicas no actúan en el vacío, sino que, por el contrario, se relacionan e interactúan con otros sistemas en los cuales es necesario situarlas para mejor comprenderlas; y propone un modelo que sitúa las creencias epistemológicas bajo la influencia de las perspectivas culturales e interactuando con otros sistemas como la autorregulación del aprendizaje y el rendimiento escolar (Schommer-Aikins, 2004).

El rol del contexto se imbrica y ha sido confundido con el problema de la generalidad-especificidad de las creencias epistemológicas (Limón, 2006b). En la investigación en epistemología personal, el término contexto ha sido usado al menos en cuatro sentidos diferentes: el dominio o disciplina del conocimiento al que las creencias aluden ( $p$. e., Buehl, Alexander \& Murphy, 2002; Schommer \& Walker, 1995); variables específicas del contexto social a las cuales se les atribuye alguna influencia sobre las creencias epistemológicas, como la educación de los padres o el acceso a recursos de información (p. e., Hall, Chiarello \& Edmonson, 1996; Schommer, 1993); el marco sociocultural amplio en que los participantes se desenvuelven (p. e., Alexander, Murphy, Guan \& Murphy, 1998; Buehl \& Alexander, 2006; Chan, 2002, 2003; Chan \& Elliot, 2002; Muis, Bendixen \& Haerle, 2006); y las características situacionales específicas (p. e., Hammer \& Elby, 2002; Hofer, 2004b; Louca, Elby, Hammer \& Kagey, 2004).

Considerando esta diversidad de usos, es indispensable establecer algunas precisiones. Cuando se le pide a una persona manifestar su grado de acuerdo o desacuerdo con una afirmación como "En matemáticas, el conocimiento sólo puede ser verdadero o falso" o "En la escuela, lo que dice el profesor es la verdad", el contexto es establecido en la misma afirmación, ya que es parte del contenido de la creencia misma. Es decir, la pregunta es 
acerca de una creencia referida a una disciplina, una situación o un contexto específico.

A este tipo de contexto al que la creencia hace alusión en sí misma, se le llamará en lo sucesivo "contexto de referencia". Limón (2006b) y Hofer (2006) advierten respecto de otra distinción, señalando que las creencias se manifiestan explícitamente en situaciones específicas, siendo afectadas por las características de la situación; en este sentido, el contexto provee las claves para expresar determinadas creencias y no otras (Hammer \& Elby, 2002; Louca, Elby, Hammer \& Kagey, 2004). A estas características de la situación en que las creencias son expresadas o manifestadas, se le llamará "contexto de realización".

Otra distinción útil ha sido planteada por Bernstein (1990). Desde la sociolingüística, el autor distingue entre contextos especializados y no especializados. Un contexto comunicacional especializado es un contexto fuertemente clasificado, en que las reglas de interacción están estrechamente ligadas a la situación; es decir, tiene roles y reglas de actuación claramente definidos, y requiere que los participantes tengan los códigos lingüísticos necesarios para reconocerlos y actuar en consecuencia. Careciendo de tales códigos, los participantes no reconocerán el carácter especializado de la situación y responderán con comportamientos no apropiados para ese carácter. De acuerdo a Bernstein, la escuela es un contexto altamente especializado, lo que concuerda con lo planteado por Alexander y sus colegas (Alexander \& Murphy, 1998; Buehl \& Alexander, 2006).

En este estudio, se intenta determinar si las creencias epistemológicas difieren cuando se refieren a los distintos niveles de creencias -general, académico o disciplinar- y si esta influencia se relaciona con la naturaleza especializada o no del contexto de referencia, así como con la familiariedad de los participantes con dicho contexto, desde el modelo del desarrollo de la comprensión epistemológica propuesto por Kuhn y sus colegas (Kuhn, 2001; Kuhn, Cheney \& Weinstock, 2000; Kuhn \& Weinstock, 2002).

La comprensión epistemológica se logra coordinando progresivamente los aspectos objetivos y subjetivos del conocimiento. En una primera instancia, llamada absolutismo, las afirmaciones son entendidas como hechos que pueden ser correctos o incorrectos, lo que se puede determinar directamente a partir de su comparación con la realidad. En una segunda instancia, llamada multiperspectivismo, ${ }^{1}$ las afirmaciones son asumidas como meras opiniones sostenidas por cada persona en función de sus propios criterios, ya que el conocimiento se entiende como una construcción personal no reductible a ninguna realidad concreta. La comprensión epistemológica se concreta al coordinarse ambos aspectos del conocimiento, objetivo y subjetivo, en la instancia evaluadora, en que el conocimiento es asumido como una construcción humana incierta y no sujeta a contrastación directa con la realidad, pero sí a criterios de argumentación y evidencia, a partir de los cuales las afirmaciones pueden ser evaluadas.

Específicamente, se hipotetizó que el nivel de comprensión epistemológica expresado en creencias epistemológicas de los distintos niveles propuestos -general, académico y disciplinar- sería distinto, y que este efecto variaría como resultado de la influencia del tipo de contexto al que refieren las creencias -especializado o no especializado-y del grado de familiariedad o proximidad de los participantes con el contexto especializado.

\section{Metodología}

\section{Diseño}

Se implementó un diseño cuasiexperimental mixto, con cuatro grupos independientes según proximidad al contexto de referencia especializado, y medidas repetidas de comprensión epistemológica para cada contexto de referencia (especializado y no especializado), en cada nivel de generalidad (general, académico y disciplinar).

1 Se ha preferido "multiperspectivismo" para traducir el término "multiplist" utilizado en el original en inglés, ya que, no teniendo ese término una traducción literal exacta, multiperspectivismo parece recoger de manera apropiada el sentido del concepto. 


\section{Participantes}

El estudio contó con 340 participantes, divididos en 4 grupos de igual tamaño $(n=85)$, cada grupo reflejando un grado distinto de proximidad con el contexto especializado utilizado en el instrumento -el contexto pedagógico: profesores de enseñanza secundaria, estudiantes de pedagogía, estudiantes de carreras no pedagógicas (de las áreas de salud e ingeniería) y apoderados de alumnos de enseñanza secundaria, residentes en cuatro ciudades chilenas de tamaño mediano (160.000 a 260.000 habitantes). Los participantes fueron seleccionados por conveniencia para cumplir con el criterio de conformación de cada uno de los grupos, e invitados a participar como voluntarios en la investigación.

\section{Materiales}

El procedimiento de papel y lápiz desarrollado por Kuhn y su equipo, cuya validez al compararlo con una medición en profundidad ha mostrado niveles satisfactorios (Kuhn \& Weinstock, 2002), fue adaptado para evaluar la comprensión epistemológica de acuerdo a los objetivos de este estudio. El procedimiento original implica plantear una situación de desacuerdo entre dos personas hipotéticas, y luego consultar si sólo uno puede estar en lo correcto o si ambos pueden estarlo (alternativas que distinguen una comprensión absolutistadualista de las multiperspectivista y evaluadora). Si la respuesta corresponde a la segunda opción, se plantea una segunda pregunta, en relación a si, pudiendo los dos personajes hipotéticos tener razón, los planteamientos de ambos pudieran ser igualmente válidos o si podría determinarse que uno pudiera ser más acertado que el otro (alternativas que distinguen entre una comprensión multiperspectivista y una comprensión evaluadora).

Dos modificaciones fueron introducidas en este procedimiento. La primera, que ambas preguntas se redujeron a una sola con tres alternativas de respuesta; la segunda, que se introdujeron ítems equivalentes en contextos de referencia diferentes. Inicialmente, se desarrolló un conjunto de 15 ítems referidos a las diferencias de opinión entre dos profe- sores en un contexto escolar, 5 referidos a cada uno de los tres niveles de creencias epistemológicas - general, académico y disciplinar-. Para cada uno de estos ítems, se desarrolló luego un ítem equivalente, pero referido a una diferencia de opinión entre dos amigos en un contexto social informal. En la Figura 1 se muestra un ítem de ejemplo.

Debido al diseño de investigación, que involucra medidas repetidas, interesaba contar con buen nivel de confiabilidad en aplicaciones sucesivas, por lo que estos 30 ítems fueron aplicados dos veces a una muestra piloto de 60 estudiantes, con una semana de intervalo, y se calculó el porcentaje de acuerdo entre ambas aplicaciones para cada ítem. Los 6 ítems que tuvieron los mejores niveles de acuerdo en ambos contextos de referencia fueron retenidos, configurándose un instrumento con 12 ítems, 6 en cada contexto de referencia y 4 en cada nivel de creencias. Esta nueva versión fue aplicada dos veces a otra muestra piloto de 68 estudiantes, esta vez en forma inmediata, con una tarea distractora entre una y otra aplicación (completar un formulario con datos demográficos). Las respuestas a cada ítem fueron luego codificadas con 0,1 y 2 correspondiendo a los niveles absolutista, multiperspectivista y evaluador de comprensión epistemológica, respectivamente. Los puntajes de los seis ítems de cada contexto de referencia fueron promediados, por lo que la puntuación global para cada contexto podía variar entre 0 -las seis respuestas en nivel absolutista- y 2 -las seis respuestas en nivel evaluador-. El acuerdo test-retest para cada ítem individual varió entre $77 \%$ y $94 \%$, con una media de $84.2 \%$, y la correlación entre las puntuaciones globales fueron $\mathrm{r}=.70$ para el contexto especializado y $\mathrm{r}=.83$ para el contexto no especializado. En todos los procedimientos, los ítems fueron aplicados en forma contrabalanceada: la mitad de los participantes contestó primero los ítems referidos al contexto especializado, y viceversa.

\section{Procedimiento}

Se contactó a los participantes durante actividades grupales habituales, como reuniones o clases. Luego de una breve explicación acerca de la investiga- 


\section{Figura 1}

Ejemplo de ítems alternativos y equivalentes del instrumento, referidos a un contexto especializado (pedagógico) y a un contexto no especializado (social-informal).

\section{A. Contexto especializado (situación pedagógica)}

\begin{tabular}{|c|c|c|c|}
\hline \multirow{2}{*}{$\begin{array}{l}\text { Claudio y Nicolás son dos profesores de la misma escuela que } \\
\text { piensan distinto respecto de muchas cosas. A continuación, } \\
\text { le presentamos algunas situaciones en que no han estado de } \\
\text { acuerdo, y le pedimos su opinión al respecto. Cada situación es } \\
\text { distinta de las demás, por lo que le solicitamos que las considere } \\
\text { cada una por separado, independientemente de su respuesta a } \\
\text { las otras situaciones. }\end{array}$} & \multicolumn{3}{|c|}{ En este caso: } \\
\hline & $\begin{array}{l}\text { Sólo uno } \\
\text { puede estar } \\
\text { en lo correc- } \\
\text { to. }\end{array}$ & $\begin{array}{l}\text { Ambos podrían } \\
\text { tener razones } \\
\text { igualmente } \\
\text { válidas o co- } \\
\text { rrectas. }\end{array}$ & $\begin{array}{l}\text { Ambos podrían te- } \\
\text { ner razón, pero uno } \\
\text { podría tener mejores } \\
\text { o más válidas razo- } \\
\text { nes que el otro. }\end{array}$ \\
\hline $\begin{array}{l}\text { En un consejo técnico en que comparaban los textos escolares } \\
\text { que usaban distintos profesores, Nicolás prefirió la diagrama- } \\
\text { ción, las ilustraciones y el colorido presentado por una editorial, } \\
\text { mientras Claudio prefirió el formato escogido por una editorial } \\
\text { distinta. }\end{array}$ & & & \\
\hline
\end{tabular}

B. Contexto no especializado (situación social-informal)

\begin{tabular}{|c|c|c|c|}
\hline \multirow{2}{*}{$\begin{array}{l}\text { Álvaro y Alberto son dos amigos, vecinos del mismo barrio, que } \\
\text { piensan distinto respecto de muchas cosas. A continuación, } \\
\text { le presentamos algunas situaciones en que no han estado de } \\
\text { acuerdo, y le pedimos su opinión al respecto. Cada situación es } \\
\text { distinta de las demás, por lo que le solicitamos que las considere } \\
\text { cada una por separado, independientemente de su respuesta a } \\
\text { las otras situaciones. }\end{array}$} & \multicolumn{3}{|c|}{ En este caso: } \\
\hline & $\begin{array}{l}\text { Sólo uno } \\
\text { puede estar } \\
\text { en lo correc- } \\
\text { to. }\end{array}$ & $\begin{array}{l}\text { Ambos podrían } \\
\text { tener razones } \\
\text { igualmente } \\
\text { válidas o co- } \\
\text { rrectas. }\end{array}$ & $\begin{array}{l}\text { Ambos podrían te- } \\
\text { ner razón, pero uno } \\
\text { podría tener mejores } \\
\text { o más válidas razo- } \\
\text { nes que el otro. }\end{array}$ \\
\hline $\begin{array}{l}\text { Comparando textos escolares en una feria de libros a la que } \\
\text { fueron con sus hijos, Alberto prefirió la diagramación, las ilus- } \\
\text { traciones y el colorido presentado por una editorial, mientras } \\
\text { Álvaro prefirió el formato escogido por una editorial distinta. }\end{array}$ & & & \\
\hline
\end{tabular}

Fuente: elaboración propia.

ción, se les solicitó la colaboración, expresada en un formulario de consentimiento. A continuación se les aplicó el instrumento, en dos formas contrabalanceadas según el contexto de referencia. Todos los datos fueron recogidos en un período de cuatro meses.

\section{Análisis de los datos}

Para cada participante, se obtuvieron nueve puntuaciones calculando los promedios de sus respuestas a cada subconjunto de ítems: nivel general de creencias epistemológicas en contexto de referencia no especializado y en contexto de referencia especializado; nivel académico de creencias epistemológicas en contexto de referencia no especializado y en contexto de referencia especiali- zado; nivel disciplinar de creencias epistemológicas en contexto de referencia no especializado y en contexto de referencia especializado; y creencias epistemológicas en cada nivel, combinando ambos contextos. Dado que la verificación de las hipótesis implica comparar diferencias entre las medias de los distintos niveles de creencias entre contextos (intrasujetos) y entre diferentes proximidades de los participantes al contexto especializado (intersujetos), se realizó análisis de varianza (ANOVA) de medidas repetidas separadamente para cada serie (contexto especializado, contexto no especializado y contexto combinado), con la variable nivel de creencias (tres niveles: general, académico, disciplinar) como factor intrasujetos y la variable proximidad al contexto especializado (cuatro niveles) como factor entre sujetos. 


\section{Resultados}

En la Tabla 1 se muestran las medias y las desviaciones típicas en la comprensión epistemológica expresada en creencias referidas a distintos contextos y niveles de generalidad, según proximidad de los participantes al contexto pedagógico.

En una primera inspección de los resultados, lo más llamativo es la regularidad del patrón que se encuentra. En todos los grupos, excepto en el de apoderados, la comprensión epistemológica se presenta más alta en las creencias de nivel general, seguida de aquellas en el nivel académico y luego, siendo el más bajo, las del nivel disciplinar, tanto en el contexto social-informal como en el pedagógico. En los apoderados, en cambio, ocurre casi exactamente lo contrario: la comprensión epistemológica aparece más baja en las creencias del nivel general, para ser seguida por los niveles académico y disciplinar, ambas en el mismo nivel o en ese orden ascendente. Aunque sólo algunas de estas diferencias son estadísticamente significativas, el patrón no puede dejar de ser notado.

Los análisis estadísticos revelan claramente estas diferencias entre los niveles de creencias epistemológicas. El ANOVA de medidas repetidas considerando ambos contextos de referencia (no especializado y especializado) combinados, mostró que el efecto del nivel de las creencias fue significativo $(F=6.233, g l=2, p<.01)$, y que este efecto interactúa con la proximidad de los participantes al contexto especializado $(F=6.369, g l=6, p<0.001)$. Las pruebas de contrastes intrasujetos muestran que ambos efectos son significativos entre los niveles general y académico $(F=10.153, g l=1, p<.005$ y $F=9.834, g l=3, p<.001$, respectivamente), pero no entre éste y el disciplinar. La proximidad de los participantes al contexto especializado, por su parte, tiene un efecto propio sobre la comprensión epistemológica $(F=3.420, g l=3, p<.05)$.

En el contexto no especializado, el ANOVA mostró que el efecto intrasujetos del nivel de las

\section{TABLA 1}

Nivel de comprensión epistemológica referida a distintos contextos y niveles de generalidad, según proximidad de los participantes al contexto pedagógico (Medias y Desviaciones Típicas).

\begin{tabular}{|c|c|c|c|c|c|c|c|c|c|}
\hline \multirow{4}{*}{$\begin{array}{c}\text { Grupo } \\
\text { (Proximidad } \\
\text { al contexto } \\
\text { pedagógico) }\end{array}$} & \multicolumn{9}{|c|}{ Contexto de referencia } \\
\hline & \multirow{2}{*}{\multicolumn{3}{|c|}{$\begin{array}{c}\begin{array}{c}\text { No especializado } \\
\text { (social-informal) }\end{array} \\
\text { Nivel de generalidad }\end{array}$}} & \multirow{2}{*}{\multicolumn{3}{|c|}{$\begin{array}{c}\begin{array}{c}\text { Especializado } \\
\text { (formal-pedagógico) }\end{array} \\
\text { Nivel de generalidad }\end{array}$}} & \multicolumn{3}{|c|}{ Combinado } \\
\hline & & & & & & & \multicolumn{3}{|c|}{ Nivel de generalidad } \\
\hline & General & $\begin{array}{l}\text { Acadé- } \\
\text { mico }\end{array}$ & $\begin{array}{l}\text { Discipli- } \\
\text { nar }\end{array}$ & General & $\begin{array}{l}\text { Acadé- } \\
\text { mico }\end{array}$ & $\begin{array}{l}\text { Disci- } \\
\text { plinar }\end{array}$ & General & $\begin{array}{l}\text { Acadé- } \\
\text { mico }\end{array}$ & $\begin{array}{l}\text { Discipli- } \\
\text { nar }\end{array}$ \\
\hline Profesor & $\begin{array}{l}1.22 \\
(.460)\end{array}$ & $\begin{array}{l}1.16 \\
(.312)\end{array}$ & $\begin{array}{l}1.11 \\
(.282)\end{array}$ & $\begin{array}{l}1.28 \\
(.520)\end{array}$ & $\begin{array}{l}1.17 \\
(.304)\end{array}$ & $\begin{array}{l}1.15 \\
(.318)\end{array}$ & $\begin{array}{l}1.25 \\
(.437)\end{array}$ & $\begin{array}{c}1.17 \\
(.263)\end{array}$ & $\begin{array}{c}1.13 \\
(.260)\end{array}$ \\
\hline $\begin{array}{l}\text { Estudiante } \\
\text { Pedagogía }\end{array}$ & $\begin{array}{c}1.33 \\
(.374)\end{array}$ & $\begin{array}{l}1.20 \\
(.338)\end{array}$ & $\begin{array}{c}1.12 \\
(.252)\end{array}$ & $\begin{array}{l}1.31 \\
(.408)\end{array}$ & $\begin{array}{c}1.24 \\
(.367)\end{array}$ & $\begin{array}{c}1.11 \\
(.257)\end{array}$ & $\begin{array}{l}1.32 \\
(.363)\end{array}$ & $\begin{array}{c}1.22 \\
(.300)\end{array}$ & $\begin{array}{c}1.11 \\
(.220)\end{array}$ \\
\hline $\begin{array}{l}\text { Estudiante otra } \\
\text { discip. }\end{array}$ & $\begin{array}{c}1.32 \\
(.413)\end{array}$ & $\begin{array}{l}1.18 \\
(.342)\end{array}$ & $\begin{array}{c}1.15 \\
(.307)\end{array}$ & $\begin{array}{c}1.31 \\
(.512)\end{array}$ & $\begin{array}{l}1.25 \\
(.375)\end{array}$ & $\begin{array}{c}1.11 \\
(.282)\end{array}$ & $\begin{array}{c}1.31 \\
(.409)\end{array}$ & $\begin{array}{c}1.21 \\
(.314)\end{array}$ & $\begin{array}{c}1.13 \\
(.263)\end{array}$ \\
\hline Apoderado & $\begin{array}{l}0.98 \\
(.615)\end{array}$ & $\begin{array}{l}1.16 \\
(.395)\end{array}$ & $\begin{array}{c}1.16 \\
(.389)\end{array}$ & $\begin{array}{c}1.05 \\
(.577)\end{array}$ & $\begin{array}{l}1.18 \\
(.413)\end{array}$ & $\begin{array}{c}1.20 \\
(.372)\end{array}$ & $\begin{array}{c}1.02 \\
(.547)\end{array}$ & $\begin{array}{c}1.17 \\
(.339)\end{array}$ & $\begin{array}{c}1.18 \\
(.339)\end{array}$ \\
\hline Total & $\begin{array}{c}1.21 \\
(.493)\end{array}$ & $\begin{array}{l}1.18 \\
(.347)\end{array}$ & $\begin{array}{c}1.14 \\
(.311)\end{array}$ & $\begin{array}{c}1.24 \\
(.517)\end{array}$ & $\begin{array}{c}1.21 \\
(.367)\end{array}$ & $\begin{array}{l}1.14 \\
(.311)\end{array}$ & $\begin{array}{l}1.23 \\
(.459)\end{array}$ & $\begin{array}{c}1.19 \\
(.305)\end{array}$ & $\begin{array}{c}1.14 \\
(.274)\end{array}$ \\
\hline
\end{tabular}

Fuente: elaboración propia. 
creencias fue significativo $(F=4.031, g l=2, p<.05)$ y que la interacción de esta variable con el nivel de proximidad de los sujetos al contexto especializado también lo era $(F=5.943, g l=6, p<.001)$. Estos efectos son significativos entre los niveles general y académico $(F=6.787, g l=1, p<.05$ y $F=8.655$, $g l=3, p<.001$ ), pero no entre el nivel académico y el disciplinar. El efecto de la proximidad al contexto especializado por sí mismo es también significativo $(F=4.174, g l=3, p<.01)$.

En el contexto especializado, el efecto intrasujetos del nivel de las creencias y su interacción con la proximidad de los participantes al contexto especializado fueron también significativos $(F=5.602, g l=2, p<.01$ y $F=4.351, g l=6, p<.005$, respectivamente), y estos efectos también resultan significativos entre los niveles general y académico de creencias $(F=9.388, g l=1, p<.005$ y $F=7.012$, $g l=3, p<.001$, respectivamente) pero no entre los niveles académico y disciplinar. El efecto de la proximidad al contexto especializado por sí mismo, sin embargo, no resultó significativo en este caso.

\section{Discusión}

Los resultados muestran claramente que hay diferencias en el nivel de comprensión epistemológica manifestado en las creencias de nivel general, académico y disciplinar. Esto es concordante con las conclusiones de Limón (2006b) y Hofer (2006), de que las creencias epistemológicas tienen tanto carácter general como específico, y apoya decididamente los modelos de Buehl y Alexander (2001, 2006) y de Muis, Bendixen y Haerle (2006), que reconocen diferentes niveles de creencias epistemológicas relacionados entre ellos, pero cada uno con su propia especificidad. El hecho de que el nivel general se distinga claramente de los niveles académico y disciplinar pero estos dos últimos no se diferencien entre sí, parece estar más de acuerdo con el modelo anidado de Buehl y Alexander (2006), que distingue entre las creencias generales y disciplinares, asimilando éstas al nivel académico, que con el propuesto por Muis, Bendixen y Haerle (2006), en que se mantiene el nivel académico como un nivel específico distinto del disciplinar.
Sin embargo, en ambos modelos se reconoce una progresión de lo general a lo particular con el desarrollo de la escolarización, y se esperaría, de acuerdo a ambos modelos, que el nivel general fuera predominante en sujetos menores y con baja escolarización, para ir avanzando hacia una diferenciación de las creencias académicas o disciplinares a medida que la escolarización progresa. Los participantes en este estudio fueron jóvenes y adultos con escolaridad avanzada, la mayor parte de ellos cursando estudios universitarios o profesionales en ejercicio; de acuerdo a ambos modelos, se esperaría en ellos un predominio o asimilación de las creencias académicas y disciplinares.

De acuerdo a lo esperado, las diferencias entre estos niveles son afectadas por el tipo de contexto al que las creencias hacen referencia y por la proximidad o familiariedad de los participantes con el tipo de contexto. Claramente, el comportamiento de los grupos con vinculación directa al contexto pedagógico -profesores, alumnos de pedagogía y alumnos universitarios de otras carreras-muestran una tendencia distinta a los apoderados, cuya relación con este contexto es indirecta o más lejana.

Sin embargo, esta interacción ocurrió tanto en las creencias referidas al contexto especializado como al no especializado, contrariando las expectativas de que la interacción se manifestara cuando las creencias se referían al contexto especializado, pero no cuando lo hacían al contexto no especializado. De la misma manera, el efecto se explica principalmente por las diferencias en las creencias del nivel general, contrariamente a lo esperado, que correspondía a mayor variación en los niveles académico o disciplinar.

En el mismo sentido apunta el hecho de que el efecto intersujetos fue significativo en el caso de las creencias referidas al contexto no especializado, pero no en el caso de las referidas al contexto especializado. Es decir, los grupos de participantes muestran diferencias en el nivel de comprensión epistemológica justamente en relación al contexto en el que no tienen diferencias entre ellos, es decir, el contexto informal no especializado, pero no en relación al contexto con el cual tienen distintos grados de familiariedad, es decir, el contexto pedagógico. 
En conjunto, los resultados permiten reconocer con claridad dos niveles de creencias epistemológicas, uno general y otro académico o disciplinar, tal como ha sido postulado en los recientes modelos integradores de la epistemología personal (Buehl \& Alexander, 2006; Muis, Bendixen \& Haerle, 2006). Asimismo, se puede afirmar que el contexto de referencia y la familiariedad con el contexto influyen en el nivel de comprensión epistemológica manifestado a través de esas creencias, lo que es también concordante con lo planteado por estos modelos, que asignan un importante rol al contexto.

Sin embargo, el modo en que esta influencia se ha dado en este estudio no corresponde a lo esperado. Una explicación posible para esto es que el contexto especializado escogido en este caso, el contexto pedagógico, tiene un carácter normativo muy fuerte incluso sobre quienes no están actualmente cercanos a dicho contexto, lo que pudiera haber afectado a todos los participantes, imponiendo restricciones a sus posibilidades de respuesta haciendo que éstas resultaran más uniformes. Para explorar esto, sería conveniente incorporar participantes con grados efectivamente menores de experiencia escolar.

Una explicación alternativa para este resultado tiene que ver con la capacidad del instrumento para marcar las diferencias entre contextos en cada nivel. Si bien la situación que marcaba las diferencias era la misma para los ítems de los tres niveles de generalidad (general, académico y disciplinar), ésta se enunciaba al comienzo del instrumento, tanto para la situación de contexto especializado como para la de contexto no especializado. Luego, se presentaban los seis ítems correspondientes al contexto, en el mismo orden en cada caso: primero los dos generales, luego los dos académicos y finalmente los dos disciplinares. Esto podría hacer que el efecto de la situación que diferenciaba los contextos fuera haciéndose menor a medida que se avanzaba en responder los ítems, influyendo en el resultado, como ha sido señalado respecto de otros estudios que han introducido modificaciones de este tipo en el procedimiento de evaluación, incluyendo instrucciones o acotaciones para establecer diferencias en algunas partes del instru- mento pero no en cada ítem (Buehl \& Alexander, 2001).

Los resultados de este trabajo muestran que es necesario y que puede resultar muy fructífero realizar estudios más específicos y precisos acerca de la relación entre el contexto y la epistemología personal, tanto desde el punto de vista conceptual como desde el punto de vista empírico, para lo cual las distinciones aquí esbozadas pueden resultar prometedoras. Precisar teórica y empíricamente el concepto de contexto puede ayudar a establecer sus relaciones con las creencias epistemológicas, orientando eventuales intervenciones sobre el contexto para facilitar el cambio de creencias epistemológicas tanto en profesores como en estudiantes, lo que sería un aporte a los esfuerzos por hacerlos más proclives a la innovación curricular y metodológica. Asimismo, ello podría contribuir a modificar las prácticas de los profesores, ya que, aunque la relación entre creencias y prácticas ha sido discutida y no está claramente establecida (Calderhead, 1996; Chan \& Cobb, 2002; Derby, 2002; Elliot, 2002; Hiebert et al., 2003; Lortie, 1975; Pape \& Hoy, 2002; Rodríguez \& López, 2006; Schraw \& Olafson, 2006; Wilcox-Herzog, 2002), hay evidencias en tal sentido (Cannobbio \& Orellana, 2006; Cornett, Yeotis \& Terwilliger, 1990; Hashweh, 1996; Hogan, 1999; Short \& Short, 1989; Wilson \& Wineburg, 1991 citado en Calderhead, 1996). Siendo la práctica de los profesores un elemento que ejerce influencia directa e indirecta sobre la motivación de los estudiantes (Alonso \& López, 1999; Covington, 2000; Moneo \& Rodríguez, 2000), la construcción de aprendizajes significativos (Collins et al., 1989 citado en Shuell, 1996), y el cambio conceptual (Alonso-Tapia, 2002), entre otros, no resulta trivial determinar cualquier factor que pudiera actuar sobre ella.

\section{Referencias}

Alexander, P. A. \& Dochy, F. J. R. C. (1994). Adults' views about knowing and believing. En R. Garner \& P. A. Alexander (Eds.), Beliefs about text and instruction with text (pp. 223-244). Hillsdale, NJ: Lawrence Erlbaum Associates, Inc. 
Alexander, P. A. \& Murphy, P. K. (1998). The research base for APA's learner-centered principles. In N. M. Lambert \& B. L. McCombs (Eds.), Issues in school reform: A sampler of psychological perspectives on learner-centered school (pp. 25-60), Washington DC: The American Psychological Association,

Alexander, P. A., Murphy, P. K., Guan, J. \& Murphy, P. (1998). How students and teachers in Singapore and the United States conceptualize knowledge and beliefs: Positioning learning within epistemological frameworks. Learning and Instruction, 8(2), 97-116.

Alonso, J. \& López, G. (1999). Efectos motivacionales de las actividades docentes en función de las motivaciones de los alumnos. En I. Pozo \& C. Monereo (Eds.), El aprendizaje estratégico: enseñar a aprender desde el currículo (pp. 35-57). Madrid: Santillana.

Alonso-Tapia, J. (2002). Knowledge assessment and conceptual understanding. En M. Limón \& L. Mason (Eds.), Reconsidering Conceptual Change (pp. 389-413). Dordrecht: Kluwer Academic Publishers.

Bendixen, L. \& Rule, D. (2004). An integrative approach to personal epistemology: A guiding model. Educational Psychologist, 39(1), 69-80.

Bernstein, B. (1990). The structuring of pedagogic discourse, Vol. IV. Class, codes and control. London: Routledge.

Brownlee, J. \& Berthelsen, D. (2006). Personal epistemology and relational pedagogy in early childhood teacher education programs. Early Years. Journal of International Research $\mathcal{E}$ Development, 26(1), 17-29.

Buehl, M. \& Alexander, P. (2001). Beliefs bout academic knowledge. Educational Psychology Review, 13 (4), 385-417.

Buehl, M. \& Alexander, P. (2006). Examining the dual nature of epistemological beliefs. International Journal of Educational Research, 45 (1-2), 28-42.

Buehl, M., Alexander, P. \& Murphy, P. K. (2002). Beliefs about schooled knowledge: Domain specific or domain general? Contemporary Educational Psychology, 27, 415-449.

Calderhead, J. (1996). Teachers: Beliefs and knowledge. En D. Berliner \& R. Calfee (Eds.), Handbook of
Educational Psychology (pp. 709-725). New York: MacMillan Library Reference USA.

Cannobbio, J. \& Orellana, O. (2006). Perspectiva pedagógica en profesores y alumnos de pedagogía. Tesis de Licenciatura no publicada, Universidad de Tarapacá, Arica, Chile.

Chan, K. W. (2002, Diciembre). Students' epistemological beliefs and approaches to learning. Paper presented at the AARE 2002 Conference, Brisbane, Australia.

Chan, K. W. (2003, noviembre). Preservice teachers' epistemological beliefs and conceptions about teaching and learning: Cultural implications for research in teacher education. Paper presented at the NZARE AARE Conference 2003, Auckland, New Zealand.

Chan, K. W. \& Elliot, R. (2002). Exploratory study of Hong Kong teacher education student's epistemological beliefs: Cultural perspectives and implications on beliefs research. Contemporary Educational Psychology, 27, 392-414.

Chandler, M., Hallett, D. \& Sokol, B. (2002). Competing claims about competing knowledge claims. In B. Hofer \& P. Pintrich (Eds.), Personal Epistemology. The psychology of beliefs about knowledge and knowing (pp. 145-168). Mahwah, NJ: Lawrence Erlbaum Associates.

Cobb, P. (2002). Epistemological world views, subject matter contexts, and the institutional setting of teaching. Issues in Education, 8(2), 149-158.

Cornett, J., Yeotis, C. \& Terwilliger, L. (1990). Teacher personal practical theories and their influence upon teacher curricular and instructional actions: A case study of secondary science teacher. Science Education, 74(5), 517-529.

Covington, M. (2000). Goal theory, motivation and school achievement: An integrative review. Annual Review of Psychology, 51, 171-200.

Delors, J. (1996). La educación o la utopía necesaria. En J. Delors (Ed.), La Educación encierra un tesoro. (Informe a la UNESCO de la Comisión Internacional sobre la educación en el siglo XXI. Compendio. Ediciones UNESCO). Disponible en, http://www. unesco.org/delors/delors_s.pdf

Derby, S. (2002). Naive teacher education + naive assessment $=$ naive teacher epistemologies: $\mathrm{A}$ response to Scraw and Olafson. Issues in Education, 8(2), 159-166. 
Hall, V. C., Chiarello, K. S. \& Edmondson, B. (1996). Deciding where knowledge comes from depends on where you look. Journal of Educational Psychology, 88, 305-313.

Hammer, D. \& Elby, A. (2002). On the Form of a Personal Epistemology. In B. Hofer \& P. Pintrich (Eds.), Personal Epistemology. The psychology of beliefs about knowledge and knowing (pp. 169- 190). Mahwah, NJ: Lawrence Erlbaum Associates.

Hashweh, M. (1996). Effects of science teachers' epistemological beliefs in teaching. Journal of Research in Science Teaching, 33, 47-63.

Hiebert, J., Gallimore, R., Garnier, H., Givvin, K., Hollingsworth, H., Jacobs, J. et al. (2003). Teaching Mathematics in seven countries: Results from the TIMSS 1999 Video Study (NCES 2003013). U.S. Department of Education, National Center for Education Statistics. Washington, DC: U.S. Government Printing Office. Recuperado el 10 de mayo, 2007, de http://nces.ed.gov/ pubs2003/2003013.pdf

Hofer, B. K. (2002). Personal epistemology as a psychological and educational construct: An introduction. In B. Hofer \& P. Pintrich (Eds.), Personal Epistemology. The psychology of beliefs about knowledge and knowing (pp. 3-14). Mahwah, NJ: Lawrence Erlbaum Associates.

Hofer, B. K. (2004a). Introduction: Paradigmatic approaches to personal epistemology, Educational Psychologist, 31(1), 1-4.

Hofer, B. (2004b). Epistemological understanding as a metacognitive process: Thinking aloud during online searching, Educational Psychologist, 31(1), $43-56$.

Hofer, B. (2006). Domain specificity of personal epistemology: Resolved questions, persistent issues, new models. International Journal of Educational Research, 45 (1-2), 85-95.

Hofer, B. K. \& Pintrich, P. R. (1997). The development of epistemological theories: Beliefs about knowledge and knowing and their relation to learning, Review of Educational Research, 67(1), 88-140.

Hofer, B. \& Pintrich, P. (2002). Personal Epistemology. The psychology of beliefs about knowledge and knowing. Mahwah, NJ: Lawrence Erlbaum Associates.
Hogan, K. (1999). Relating students' personal frameworks for science learning to their cognition in collaborative contexts. Science Education, 83, 1-32.

Kuhn, D. (2001). How do people know? Psychological Science, 12(1), 1-8.

Kuhn, D., Cheney, R. \& Weinstock, M. (2000). The development of epistemological understanding. Cognitive Development, 15, 309-328.

Kuhn, D. \& Weinstock, M. (2002). What is epistemological thinking and why does it matter? In B. Hofer \& P. Pintrich (Eds.), Personal Epistemology. The psychology of beliefs about knowledge and knowing (pp. 121-144). Mahwah, NJ: Lawrence Erlbaum Associates,

Limón, M. (2006a). Introduction. International Journal of Educational Research, 45 (1-2), 1-6.

Limón, M. (2006b). The domain generality-specificity of epistemological beliefs: A theoretical problem, a methodological problem or both? International Journal of Educational Research, 45 (1-2), 7-27.

Lortie, D. (1975). Schoolteacher: A sociological study. Chicago: University of Chicago Press.

Louca, L., Elby, A., Hammer, D. \& Kagey, T. (2004). Epistemological resources: Applying a new epistemological framework to science instruction, Educational Psychologist, 31 (1), 57-68.

Moneo, M. \& Rodríguez, C. (2000). La construcción del conocimiento y la motivación por aprender. Psicología Educativa, 6(2), 129-149.

Muis, K. R. (2004). Personal epistemology and mathematics: A critical review and synthesis of research. Review of Educational Research, 74(3), 317-377.

Muis, K. R., Bendixen, L. D. \& Haerle, F. C. (2006). Domain-generality and domain-specificity in personal epistemology research: Philosophical and empirical reflections in the development of a theoretical framework. Educational Psychology Review, 18, 3-54.

Pajares, F. (1992). Teachers' beliefs and educational research: Cleaning up a messy construct. Review of Educational Research, 62 (3), 307-332.

Pape, S. \& Hoy, A. (2002). Whilst congruence: Teacher epistemological world views in the context of modern schooling. Issues in Education, 8(2), 195-204.

Pintrich, P. R. (2002). Future challenges and directions for theory and research on personal epistemology, 
In B. Hofer \& P. Pintrich (Eds.), Personal Epistemology. The psychology of beliefs about knowledge and knowing (pp. 389-414). Mahwah, NJ: Lawrence Erlbaum Associates,

Rodríguez, D. \& López, A. (2006). ¿Cómo se articulan las concepciones epistemológicas y de aprendizaje con la práctica docente en el aula? Tres estudios de caso de profesores de secundaria. Revista Mexicana de Investigación Educativa, 11 (31), 1307-1335.

Schommer, M. (1993). Epistemological development and academic performance among secondary students. Journal of Educational Psychology, 85, 1-6.

Schommer, M. \& Walker, K. (1995). Are Epistemological Beliefs Similar Across Domains? Journal of Educational Psychology, 87(3), 424-432.

Schommer-Aikins, M. (2004). Explaining the epistemological belief system: Introducing the embedded systemic model and coordinated research approach. Educational Psychologist, 39(1), 19-30.
Schraw, G. \& Olafson, L. (2002). Teacher's epistemological world views and educational practices. Issues in Education, 8(2), 99-149.

Schraw, G. \& Sinatra, G. (2004). Epistemological development and its impact on cognition in academic domains. Contemporary Educational Psychology, 29(2), 95-102.

Short, R. \& Short, P. (1989). Teacher beliefs, perceptions of behavior problems and intervention preferences. Journal of Social Studies Research, 13(2), 28-33.

Shuell, T. (1996). Teaching and learning in a classroom context. En D. Berliner \& R. Calfee (Eds.), Handbook of Educational Psychology (pp. 726-764). Mawah, NJ: Laurence Erlbaum Associates.

Wilcox-Herzog, A. (2002). Is there a link between teachers' beliefs and behaviors? Early Education and Development, 13(1), 81-61. 